Bull. Egypt. Soc. Physiol. Sci. Vol. (42) Issue (1), 118-130

\author{
Bull. of Egyp. Soc. Physiol. Sci. \\ (Official Journal of Egyptian Society for Physiological Sciences) \\ (pISSN: 1110-0842; eISSN: 2356-9514)
}

\title{
Prevalence of Multidrug Resistant Bloodstream Bacterial Infections among Hospitalized COVID-19 Egyptian Patients and Evaluation of Predictors for Case Severity
}

\author{
Amal Ali El-Koa ${ }^{1}$, Asmaa Shaaban Sleem², Sally Mohammed El-Hefnawy ${ }^{3}$, Nehad Badr Elsaid ${ }^{4}$ \\ and Mai Mahmoud El Kalashy ${ }^{1}$ \\ ${ }^{1}$ Chest Department, Faculty of Medicine, Menoufia University, \\ ${ }^{2}$ Medical microbiology and immunology Department, Faculty of Medicine, Menoufia University, \\ ${ }^{3}$ Medical Biochemistry and Molecular Biology Department, Faculty of Medicine, Menoufia University \\ ${ }^{4}$ Public Health and Community Medicine Department, Faculty of Medicine, Menoufia University
}

Submit Date: 13 August 2021

Revise Date: 7 Sept 2021

Accept Date : 3 Oct 2021

\section{Keywords}

COVID-19

PCR

bacterial co-infection blood

\section{Abstract}

Background and Aim: Coronaviruses are dangerous human and animal pathogens. It is characterized by fast spread, starting as an epidemic through China, followed by a worldwide pandemic. This study aimed to determine the prevalence of laboratory proven blood stream bacterial co-infection in hospitalized adult COVID-19 Egyptian patients and to recognize antibiotic susceptibilities of identified pathogens together with evaluation of predictors for case severity.Methods: The study involved 142 adult COVID-19 Egyptian patients. The diagnosis was confirmed by quantitative or real time polymerase chain reaction (PCR) on nasopharyngeal swabs. For each case two blood cultures were taken after $\geq 3$ days of hospital admission. Results:About $30 \%$ of positive blood cultures showed Gram positive staphylococci while Gram negative bacilli were detected by $70 \%$ (Klebsiella ;30\%, E.coli; $20 \%$ and Pseudomonas; 20\%). Variable resistance patterns were noticed in all bacterial isolates.Blood stream infections (BSI) were identified in 40 cases $(28 \%)$. About $70 \%$ of them were males. The mean age significantly associated with BSI was $63.5 \pm 18.2$ years old. Hypertension and diabetes had high significant association with BSI by $55 \%$ and $60 \%$ respectively. Significantly $68 \%$ of mechanically ventilated COVID cases were BSI complicated. Death was the fate of $57.5 \%$ of BSI positive COVID cases significantly with low $\mathrm{O}_{2}$ saturation and prolonged hospital stay (21.6 \pm 3.52 days). Conclusion: All blood stream bacterial pathogens were multidrug resistant. Infections were detected among old aged, diabetic, hypertensive and mechanically ventilated patients. Prescribing antibiotics in COVID patients should be guided by careful clinical and laboratory assessment to improve their management and outcomes.

Corresponding author: Sally Mohammed El-Hefnawy, E-mail: doctor_sally@ rocketmail.com. SALI.ALhefnawy@med.menofia.edu.eg, ORCID: https://orcid.org/0000-0003-3090-0141 


\section{INTRODUCTION}

Coronavirus disease 2019 (COVID-19) is caused by a positive-sense, single-stranded RNA (ssRNA) transforming virus. It is genetically related to severe acute respiratory syndrome (SARS) and Middle East respiratory syndrome (MERS). Corona viruses are common causes of severe community-acquired pneumonia (1). COVID-19 is markedly infectious disease, with every case seeding more than two secondary cases. By the end of June 2021 the number of people identified with COVID-19 internationally crossed the 185 million with more than 4 million deaths since the beginning of the pandemic (2). Egypt is one of the five countries recording the highest number of cases in Africa. (3)

Clinical pictures of COVID-19 are not specific. Diagnosis requires sufficient clinical, laboratory and radiological assessment of suspected cases. Treatment protocol according to the WHO includes home isolation for mild to moderate stable cases. Critical (respiratory failure, shock, multiple organ dysfunction or failure) and severe (dyspnoea, respiratory rate $\geq 30$ breaths per min, oxygen saturation $\leq 92 \%$ ) cases need to be hospitalized. (2)

Mortality in COVID-19 cases is usually related to older age, comorbidities as (hypertension, diabetes mellitus, cardiovascular disease, chronic lung disease and cancer), higher viral load, worse respiratory failure, higher d-dimer and C-reactive protein levels, lower WBCS count and secondary infections (4).

Bloodstream infections (BSIs) represent one of the commonest and life-threatening complications in patients with severe viral infections. The absence of an effective anti-viral agent against SARS-CoV2 combined with challenges in distinguishing secondary bacterial co-infection from severe COVID-19 infection alone, has fostered the widespread usage of empirical antibiotics in the immediate management of those patients that encourage multi drugresistant secondary bacterial infections (5). We concentrated here on BSIs because these represent sure infection events, whereas difference between colonization and infection is more difficult with other specimen types (6). This study aimed to determine the prevalence of laboratory proven blood stream bacterial co-infection in adult COVID-19 Egyptian patients and to describe the demographic and laboratory characteristics of these patients and recognize antibiotic susceptibilities of identified pathogens, also to detect the impact of BSIs on the patient outcome together with evaluation of predictors for case severity.

\section{Patients and methods}

The study involved 142 adult COVID-19 patients admitted to Menoufia fever hospital, Egypt from January to April 2021. The patients were diagnosed by real time PCR on nasopharyngeal swabs. For each case two blood cultures were taken after $\geq 3$ days of admission. All patients had received empirical antibiotics in the form of a second- or third-generation cephalosporin plus azithromycin. Mortality was measured from the date of admission till the date of death. Data were assembled from hospital records including: demographics (age, sex, presence or absence of co-morbidity), and if need to be admitted in ICU or in ward. Laboratory investigations were done in the form of $\mathrm{CBC}, \mathrm{D}$ 
dimer, serum ferritin and CRP. The patients were categorized according to the previous CT presentations using a CORAD radiologic scoring system of the method previously reported by

\section{Prokop et al (7).}

\section{Ethical Approval}

The studied population was enrolled after obtaining written consent and approval from the university research ethics committee of the Menoufia Faculty of Medicine.

\section{Specimen Collection and RNA isolation}

Nasopharyngeal specimens were collected using sterile synthetic fiber swabs with thin plastic. Swabs were placed into the transport tube provided and stored at $-80^{\circ} \mathrm{C}$ till PCR analysis. RNA was extracted from samples using Qiagen QIAamp Viral RNA Mini Kit, USA according to manufacturer instructions. The concentrations of RNA were assessed using the NanoDrop ${ }^{\mathrm{TM}} 2000$ system (Thermo Scientific, USA).

cDNA synthesis and PCR amplification in one step method

The RNA extracts were kept at $-80^{\circ} \mathrm{C}$ until the reverse transcription step. After extraction, RNA was reverse transcribed to cDNA and amplified in a Real-time PCR instrument using one step method. The kit includes primers/probes that are specific for the ORF1ab gene (probe labeled with FAM) and $\mathrm{N}$ gene (probe labeled with VIC) of SARS-CoV-2. In addition, the kit also contains primers and a probe (labeled with CY5) for the human RNase $\mathrm{P}$ gene as an endogenous internal control for specimen integrity, nucleic acid isolation, amplification and detection. The reactions were performed on ice in a $25 \mu$ l total volume as follows: we added $7.5 \mu \mathrm{l}$ of RT-PCR Buffer (Tris Hydroxy Methyl Aminomethan,
Potassium chloride, Magnesium chloride, Nucleotides mix), $5 \mu$ of RT-PCR Enzyme Mix (Reverse transcriptase, RNase Inhibitor, Taq DNA polymerase), $4.0 \mu \mathrm{L}$ of reaction mix (Primers and probes of SARS-CoV-2 and RNase P), $3.5 \mu$ Lof RNase-free water and $5 \mu \mathrm{l}$ of RNA extract to each PCR well and $5 \mu \mathrm{L}$ of extract from the positive control to one reaction well. The following primers were used: RdRP gene forward primer GTGARATGGTCATGTGTGGCGG, reverse CARATGTTAAASACACTATTAGCATA, E gene forward primer ACAGGTACGTTAATAGTTAATAGCGT, reverse ATATTGCAGCAGTACGCACACA, N gene forward primer CACATTGGCACCCGCAATC, reverse GAGGAACGAGAAGAGGCTTG. The thermal cycling conditions were modified as follows: $50^{\circ} \mathrm{C}, 10 \mathrm{~min}$ for one cycle at (hold stage), $97^{\circ} \mathrm{C}$ for $1 \mathrm{~min}$ for one cycle at (second hold stage). 45 cycle each one composed of: $97^{\circ} \mathrm{C}$ for 5 s initial PCR stage, $58^{\circ} \mathrm{C}$ for $30 \mathrm{~s}, 72^{\circ} \mathrm{C}$ for $30 \mathrm{~s}$. Data were analyzed by the software accompanying the ABI 7500 real-time PCR device, V.2.0.1.

\section{Microbiological test and Blood Culture}

Blood samples were taken under complete aseptic conditions from COVID patients hospitalized $\geq 3$ days. Samples were immediately transferred to the bacteriology laboratory to be aerobically cultivated at $35-37^{\circ} \mathrm{C}$ (Becton Dickinson blood cultures instrument systems) for a maximum of 5 days. All clinically significant bacterial isolates were recognized by standard bacteriological methods (all contaminants were excluded). Antimicrobial susceptibility testing was performed and assessed by the standard disc diffusion methods. Also Multi-drug resistant (MDR) strains were 
identified. All methods were done according to Clinical \& Laboratory Standards Institute (CLSI) guidelines (8).

Detection of methicillin-resistant staphylococci was done by cefoxitin disk diffusion method $(30 \mu \mathrm{g})$. Screening for ES $\beta$ Ls production was performed by disk diffusion test using ceftazidime $(30 \mu \mathrm{g})$, cefotaxime $(30 \mu \mathrm{g})$, ceftriaxone $(30 \mu \mathrm{g})$ and aztreonam $(30 \mu \mathrm{g})$. Suspected ES $\beta \mathrm{L}$ producing Gram-negative bacilli were confirmed by clavulinate combined disk test. Screening for carbapenemase production was performed by disk diffusion test. Reduced susceptibility to one or more carbapenems (meropenem and etrapenem; $10 \mu \mathrm{g})$ plus one or more of the indicator cephalosporins (cefotaxime, ceftriaxone, ceftazidime and cefoperazone) indicate carbapenemase production. Suspected carbapenemase producers were confirmed by modified carbapenem inactivation method $(\mathrm{mCIM})(8)$. (Figure 1 a\&b).

\section{Statistical analysis}

Data were collected, tabulated, statistically analyzed using an IBM personal computer with Statistical Package of Social Science (SPSS) version 19 (SPSS, Inc, Chicago, Illinois, USA) quantitative data were displayed in the form of mean, standard deviation (SD), range, and qualitative data were presented in the form of number and percent. Chi-square test $\left(\chi^{2}\right)$ and Fisher exact test was used to study association between qualitative variables, Mann-Whitney test was used for comparison between quantitative variables. Univariate and multivariate regression analysis models were used to detect independent predictors for ICU admission. Significance level was at $\mathrm{p}$ value $<0.05$.

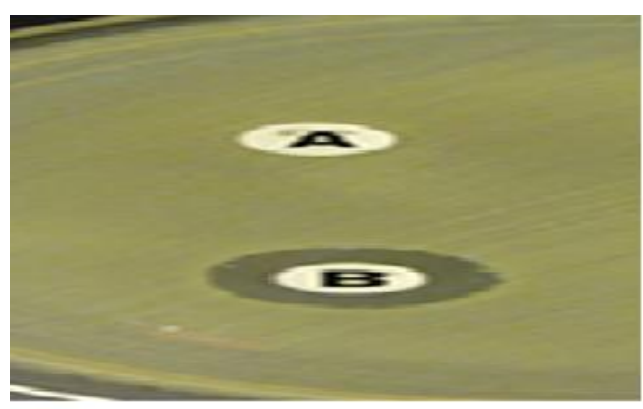

A

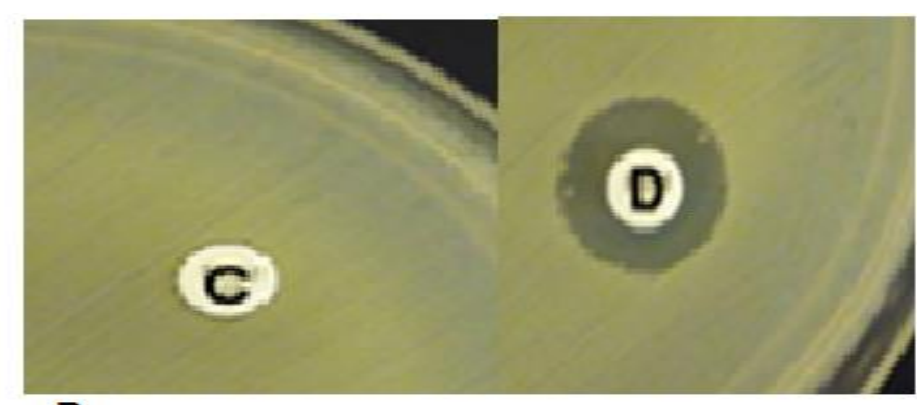

B

Figure (1): a- Detection of ESBL production using the clavulinate combined disc method) CLSI 2021). The A disc is ceftazidime (30 $\mathrm{ug})$ and the B disc is ceftazidime/clavulinic acid (30/10 ug). There was an expansion of the bacterial growth inhibition zones around B disc $>5 \mathrm{~mm}$. b- Detection of carbapenemase production using the modified carbapenem inactivation method (mCIM) (CLSI 2021). Meropenem disc (10ug) was embedded in emulsified suspension of tested strain for carbapenemase production. Then this disc was placed on plate previously inoculated with carbapenem susceptible control strain. Tested carbapenemase producers were detected by growth inhibition zones of 6-15 $\mathrm{mm}$ around C \& D meropenem discs.

\section{Results}

This study involved 142 COVID 19 PCR confirmed cases, their ages were ranged between (27 to 89 years), 82 of them $(57.7 \%)$ was male and $68(42.3 \%)$ was female gender. The most common comorbidities were diabetes mellitus (39.4\%) and hypertension (38\%). As regards the clinical findings, fever (93\%), dyspnea (90.1\%) and cough
(84.5\%) were the most common presenting symptoms while GIT symptoms (e.g., nausea, vomiting, abdominal pain and diarrhea) represented (39.4\%) (Table I).

The CT examination of the studied cases showed 2 cases (1.4\%) with CORAD I and another 2 cases $(1.4 \%)$ with CORAD II, 24 cases (16.9\%) with CORAD III, 70 cases (49.3\%) with CORAD 
IV and 44 cases (31\%) with CORAD V. The mean oxygen saturation among cases was 88.2 \pm 7.35 . The cases that needed mechanical ventilation were 50 cases $(35.2 \%)$. The passed away cases were 28 $(19.7 \%)$ cases. The mean hospital stay days were $8.86 \pm 8.30$ days (Table II).

Table III shows highly significant differences between ventilated and non-ventilated patients regarding age, dyspnea and cyanosis at their initial presentation and diabetes as a comorbidity. In contrast there were nonsignificant differences between the two groups regarding sex, other comorbidities and fever, cough and GIT symptoms as presenting symptoms.

Also, there were highly significant differences between ventilated and non-ventilated patients regarding CT scoring, mean oxygen saturation on presentation and fate of the disease.

There was a positive correlation between age and hospital stay and a negative correlation between age and $\mathrm{O}_{2}$ saturation (Table IV).

Blood stream infections (BSI) were detected in $40 / 142$ cases $(28 \%)$. About $70 \%$ were males. The mean age of $63.5 \pm 18.2$ years old was significantly associated with BSI. Hypertension and diabetes showed high significant association with BSI by $55 \%$ and $60 \%$ respectively.
Significantly $34150 \quad(68 \%)$ of mechanically ventilated cases had multi drug resistant BSI, while 6/92(6.5\%) of non ventilated cases had BSI. Eighty five percent of COVID patients with positive BSI (34/40) were mechanically ventilated of whom $88.2 \%$ (30/34) were badly deteriorated. About $30 \%$ of (40) positive blood cultures showed Gram positive staphylococci while Gram negative bacilli were detected by $70 \%$ (Klebsiella spp;30\%, E.coli ; 20\% and Pseudomonas spp; 20\%). Variable resistance patterns were detected in all bacterial isolates as shown in figure $(\mathbf{2} \mathbf{a \& b})$.

Twenty eight COVID 19 included patients in concurrent study died by $19.7 \%$ mortality rate. About $85.7 \%$ of non survivors were mechanically ventilated and needed ICU care. Death was the fate of $57.5 \%$ (23/40) of BSI positive COVID cases mainly with low $\mathrm{O} 2$ saturation and prolonged hospital stay $(21.6 \pm 3.52$ days $)$ with high significance, while only $4.9 \%(5 / 102)$ of BSI negative COVID cases died with chronic diseases (Table VI).

High CT score, O2 desaturation and secondary bacterial blood stream infection are independent predictors for need for mechanical ventilation ( $\mathrm{P}$ values $0.013,0.023$ and 0.001 respectively) as shown in table VII.

Table (I): Socio demographic and clinical data of the studied group ( $N=142)$ :

\begin{tabular}{|c|c|c|}
\hline Studied variables & No. & $\%$ \\
\hline $\begin{array}{l}\text { Age / years } \\
\text { Mean } \pm \mathrm{SD} \\
\text { Range } \\
\end{array}$ & \multicolumn{2}{|c|}{$\begin{array}{c}58.8 \pm 15.5 \\
27-89 \\
\end{array}$} \\
\hline $\begin{array}{l}\text { Sex } \\
\text { Male } \\
\text { Female }\end{array}$ & $\begin{array}{l}82 \\
60\end{array}$ & $\begin{array}{l}57.7 \\
42.3\end{array}$ \\
\hline $\begin{array}{l}\text { Co morbidities } \\
\text { Hypertension } \\
\text { Diabetes mellitus } \\
\text { Cardic disease } \\
\text { Renal disease } \\
\text { Chronic lung disease } \\
\text { Neurological }\end{array}$ & $\begin{array}{c}54 \\
56 \\
8 \\
2 \\
10 \\
7\end{array}$ & $\begin{array}{l}38.0 \\
39.4 \\
5.60 \\
1.40 \\
7.00 \\
4.90\end{array}$ \\
\hline $\begin{array}{l}\text { Clinical presentation } \\
\text { Fever } \\
\text { Cough } \\
\text { Dyspnea } \\
\text { Cyanosis } \\
\text { GIT symptoms }\end{array}$ & $\begin{array}{c}132 \\
120 \\
128 \\
62 \\
56\end{array}$ & $\begin{array}{l}93.0 \\
84.5 \\
90.1 \\
43.7 \\
39.4\end{array}$ \\
\hline
\end{tabular}


Table (II): Radiological finding, $\mathrm{O} 2$ saturation, $\mathrm{O} 2$ therapy, need for mechanical ventilation, fate and hospital stay/days among the studied group $(\mathrm{N}=142)$ :

\begin{tabular}{|c|c|c|}
\hline Studied variables & No. & $\%$ \\
\hline $\begin{array}{l}\mathbf{C T} \\
\text { CORAD I } \\
\text { CORAD II } \\
\text { CORAD III } \\
\text { CORAD IV } \\
\text { CORAD V } \\
\end{array}$ & $\begin{array}{c}2 \\
2 \\
24 \\
70 \\
44 \\
\end{array}$ & $\begin{array}{l}1.40 \\
1.40 \\
16.9 \\
49.3 \\
31.0 \\
\end{array}$ \\
\hline $\begin{array}{l}\text { O2 saturation } \\
\text { Mean } \pm \text { SD } \\
\text { Range } \\
\end{array}$ & \multicolumn{2}{|c|}{$\begin{array}{c}88.2 \pm 7.35 \\
45-98 \\
\end{array}$} \\
\hline Need for mechanical ventilation & 50 & 35.2 \\
\hline $\begin{array}{l}\text { Fate } \\
\text { Died } \\
\text { Alive }\end{array}$ & $\begin{array}{c}28 \\
114\end{array}$ & $\begin{array}{l}19.7 \\
80.3\end{array}$ \\
\hline $\begin{array}{l}\text { Hospital stay /days } \\
\text { Mean } \pm \text { SD } \\
\text { Range }\end{array}$ & \multicolumn{2}{|c|}{$\begin{array}{c}8.86 \pm 8.30 \\
3-28\end{array}$} \\
\hline
\end{tabular}

Table (III): Relation between need for mechanical ventilation and clinical, radiological parameters and outcome of the studied group $(\mathrm{N}=142)$ :

\begin{tabular}{|c|c|c|c|c|c|c|}
\hline \multirow[t]{2}{*}{ Studied variables } & \multicolumn{4}{|c|}{ need for mechanical ventilation } & \multirow[t]{2}{*}{ Test of sig. } & \multirow[t]{2}{*}{ P value } \\
\hline & \multicolumn{2}{|c|}{$\begin{array}{c}\text { Yes } \\
(\mathrm{N}=50)\end{array}$} & \multicolumn{2}{|c|}{$\begin{array}{c}\text { No } \\
(\mathrm{N}=92)\end{array}$} & & \\
\hline $\begin{array}{l}\text { Age / years } \\
\text { Mean } \pm \text { SD }\end{array}$ & \multicolumn{2}{|c|}{$64.8 \pm 18.4$} & \multicolumn{2}{|c|}{$55.5 \pm 12.7$} & $\begin{array}{c}\mathrm{U} \\
3.56\end{array}$ & $0.001 * *$ \\
\hline $\begin{array}{l}\text { Sex } \\
\text { Male } \\
\text { Female }\end{array}$ & $\begin{array}{l}30 \\
20\end{array}$ & $\begin{array}{l}60.0 \\
40.0\end{array}$ & $\begin{array}{l}52 \\
40\end{array}$ & $\begin{array}{l}56.5 \\
43.5\end{array}$ & $\begin{array}{c}X^{2} \\
0.161\end{array}$ & 0.689 \\
\hline $\begin{array}{l}\text { Co morbidities } \\
\text { Hypertension } \\
\text { Diabetes mellitus } \\
\text { Cardic disease } \\
\text { Renal disease } \\
\text { Chronic lung disease } \\
\text { Neurological }\end{array}$ & $\begin{array}{l}22 \\
26 \\
5 \\
0 \\
6 \\
4\end{array}$ & $\begin{array}{l}44.0 \\
52.0 \\
10.0 \\
0.00 \\
12.0 \\
8.00\end{array}$ & $\begin{array}{l}32 \\
30 \\
3 \\
2 \\
4 \\
3\end{array}$ & $\begin{array}{l}34.8 \\
32.6 \\
3.30 \\
2.20 \\
4.30 \\
3.30\end{array}$ & $\begin{array}{l}\mathrm{X}^{2} \\
\\
1.16 \\
5.10 \\
2.83 \\
1.10 \\
2.90 \\
1.55\end{array}$ & $\begin{array}{c}0.280 \\
\mathbf{0 . 0 2 4} * \\
0.092 \\
0.293 \\
0.088 \\
0.212\end{array}$ \\
\hline $\begin{array}{l}\text { Clinical presentation } \\
\text { Fever } \\
\text { Cough } \\
\text { Dyspnea } \\
\text { Cyanosis } \\
\text { GIT symptoms }\end{array}$ & $\begin{array}{l}48 \\
44 \\
50 \\
40 \\
15\end{array}$ & $\begin{array}{c}96.0 \\
88.0 \\
100.0 \\
80.0 \\
30.0\end{array}$ & $\begin{array}{l}84 \\
76 \\
78 \\
22 \\
41\end{array}$ & $\begin{array}{l}91.3 \\
82.6 \\
84.8 \\
23.9 \\
44.6\end{array}$ & $\begin{array}{c}\mathrm{X}^{2} \\
1.09 \\
1.91 \\
8.44 \\
41.4 \\
2.88\end{array}$ & $\begin{array}{c}0.296 \\
0.383 \\
\mathbf{0 . 0 0 2} * * \\
\mathbf{0 . 0 0 1} * * \\
0.089\end{array}$ \\
\hline $\begin{array}{l}\text { CT } \\
\text { CORAD I } \\
\text { CORAD II } \\
\text { CORAD III } \\
\text { CORAD IV } \\
\text { CORAD V }\end{array}$ & $\begin{array}{c}0 \\
0 \\
4 \\
16 \\
30\end{array}$ & $\begin{array}{l}0.00 \\
0.00 \\
8.00 \\
32.0 \\
60.0\end{array}$ & $\begin{array}{c}2 \\
2 \\
20 \\
54 \\
14\end{array}$ & $\begin{array}{l}2.20 \\
2.20 \\
21.7 \\
58.7 \\
15.2\end{array}$ & $\begin{array}{c}X^{2} \\
31.4\end{array}$ & $0.001 * *$ \\
\hline $\begin{array}{l}\text { O2 Saturation } \\
\text { Mean } \pm \text { SD }\end{array}$ & \multicolumn{2}{|c|}{$82.3 \pm 10.1$} & \multicolumn{2}{|c|}{$90.6 \pm 4.02$} & $\begin{array}{c}U \\
6.25\end{array}$ & $0.001 * *$ \\
\hline $\begin{array}{l}\text { Fate } \\
\text { Died } \\
\text { Alive }\end{array}$ & $\begin{array}{l}24 \\
26\end{array}$ & $\begin{array}{l}48.0 \\
52.0\end{array}$ & $\begin{array}{c}4 \\
88\end{array}$ & $\begin{array}{l}4.30 \\
95.7\end{array}$ & $\begin{array}{c}X^{2} \\
38.9\end{array}$ & $0.001 * *$ \\
\hline $\begin{array}{l}\text { Hospital stay /days } \\
\text { Mean } \pm \text { SD }\end{array}$ & \multicolumn{2}{|c|}{$16.1 \pm 8.86$} & \multicolumn{2}{|c|}{$4.91 \pm 4.44$} & $\begin{array}{c}\mathrm{U} \\
7.14\end{array}$ & $0.001 * *$ \\
\hline
\end{tabular}

U: Mann Whitney test \#: Fisher exact test *Significant **High significant 
Table (IV): Correlation between age / years and 02 saturation and hospital stay among the studied group $(\mathrm{N}=142)$ :

\begin{tabular}{|l|c|c|}
\hline \multirow{2}{*}{ Studied variables } & \multicolumn{2}{|c|}{ Age/ years } \\
\cline { 2 - 3 } & $\mathbf{r}$ & P value \\
\hline O2 saturation & -0.224 & $\mathbf{0 . 0 0 7} * *$ \\
\hline Hospital stay & 0.274 & $\mathbf{0 . 0 0 1} * *$ \\
\hline
\end{tabular}

Table (V): Relation between need for need for mechanical ventilation and blood culture results of the studied group $(\mathrm{N}=142)$ :

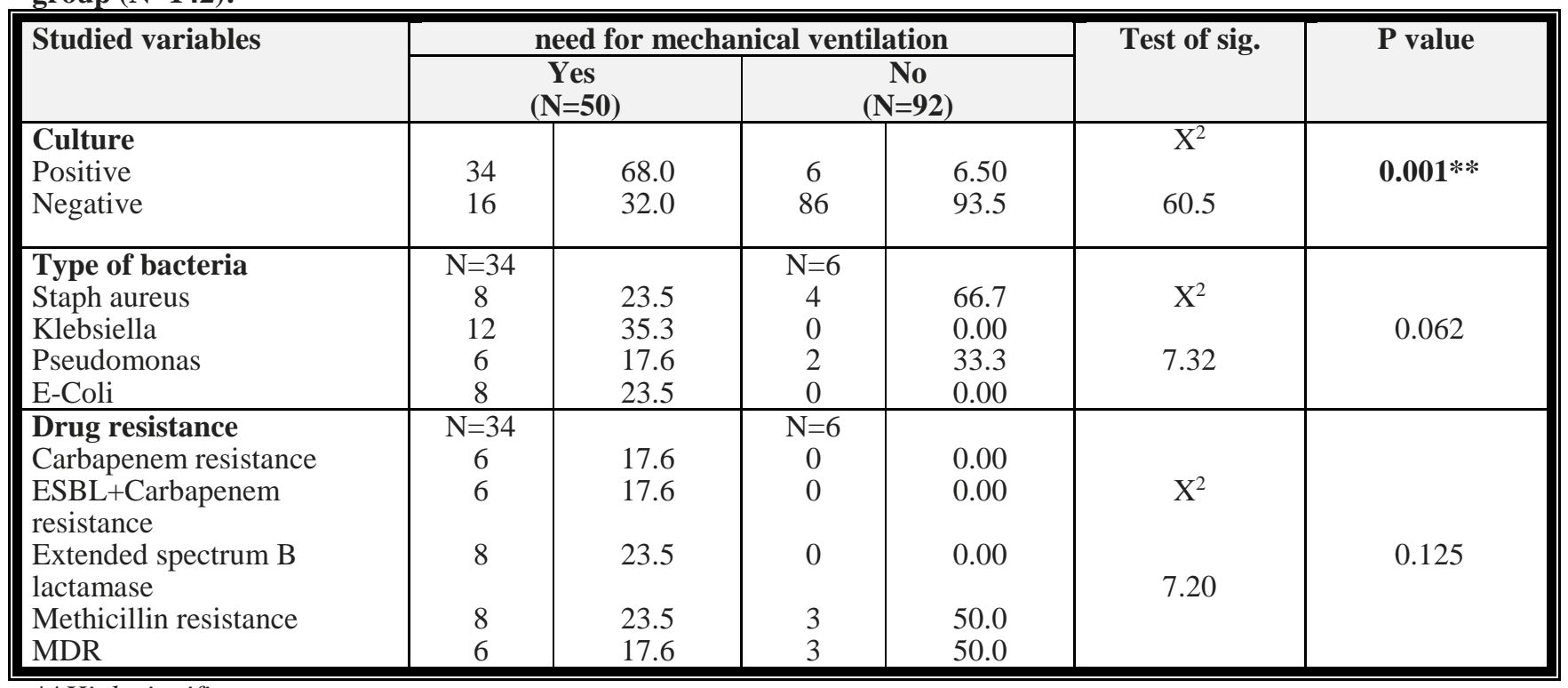

**High significant

Table (VI): Relation between blood stream infection and clinical parameter of the studied group (N=142):

\begin{tabular}{|c|c|c|c|c|c|c|}
\hline \multirow[t]{2}{*}{ Studied variables } & \multicolumn{4}{|c|}{ Blood Culture } & \multirow{2}{*}{$\begin{array}{c}\text { Test of } \\
\text { sig. }\end{array}$} & \multirow[t]{2}{*}{$P$ value } \\
\hline & \multicolumn{2}{|c|}{$\begin{array}{c}\text { Positive } \\
(\mathrm{N}=40)\end{array}$} & \multicolumn{2}{|c|}{$\begin{array}{r}\text { Negative } \\
(\mathrm{N}=102)\end{array}$} & & \\
\hline $\begin{array}{l}\text { Age / years } \\
\text { Mean } \pm \mathrm{SD}\end{array}$ & \multicolumn{2}{|c|}{$63.5 \pm 18.2$} & \multicolumn{2}{|c|}{$56.9 \pm 14.1$} & $\begin{array}{c}\mathrm{U} \\
2.23\end{array}$ & $0.026 *$ \\
\hline $\begin{array}{l}\text { Sex } \\
\text { Male } \\
\text { Female }\end{array}$ & $\begin{array}{l}28 \\
12\end{array}$ & $\begin{array}{l}70.0 \\
30.0\end{array}$ & $\begin{array}{l}54 \\
48\end{array}$ & $\begin{array}{l}52.9 \\
47.1\end{array}$ & $\begin{array}{c}\mathrm{X}^{2} \\
3.42\end{array}$ & 0.064 \\
\hline $\begin{array}{l}\text { Co morbidities } \\
\text { Hypertension } \\
\text { Diabetes mellitus } \\
\text { Cardic disease } \\
\text { Renal disease } \\
\text { Chronic lung disease } \\
\text { Neurological }\end{array}$ & $\begin{array}{l}22 \\
24 \\
3 \\
1 \\
4 \\
3\end{array}$ & $\begin{array}{l}55.0 \\
60.0 \\
7.50 \\
2.50 \\
10.0 \\
7.50\end{array}$ & $\begin{array}{c}32 \\
32 \\
5 \\
1 \\
6 \\
4\end{array}$ & $\begin{array}{l}31.4 \\
31.4 \\
4.90 \\
0.98 \\
5.90 \\
3.90\end{array}$ & $\begin{array}{c}\mathrm{X}^{2} \\
6.80 \\
9.85 \\
0.360 \# \\
0.480 \# \\
0.740 \# \\
0.790 \#\end{array}$ & $\begin{array}{c}\mathbf{0 . 0 0 9} * * \\
\mathbf{0 . 0 0 2} * * \\
0.545 \\
0.489 \\
0.388 \\
0.375\end{array}$ \\
\hline $\begin{array}{l}\text { Clinical presentation } \\
\text { Fever } \\
\text { Cough } \\
\text { Dyspnea } \\
\text { Cyanosis } \\
\text { GIT symptoms }\end{array}$ & $\begin{array}{l}38 \\
32 \\
38 \\
30 \\
16\end{array}$ & $\begin{array}{l}95.0 \\
80.0 \\
95.0 \\
75.0 \\
32.0\end{array}$ & $\begin{array}{l}94 \\
88 \\
90 \\
32 \\
40\end{array}$ & $\begin{array}{l}92.2 \\
86.3 \\
88.2 \\
31.4 \\
43.5 \\
\end{array}$ & $\begin{array}{c}\mathrm{X}^{2} \\
0.355 \# \\
0.860 \# \\
1.47 \# \\
22.2 \\
1.79 \\
\end{array}$ & $\begin{array}{c}0.725 \\
0.352 \\
0.350 \\
\mathbf{0 . 0 0 1} * * \\
0.181 \\
\end{array}$ \\
\hline $\begin{array}{l}\text { Fate } \\
\text { Died } \\
\text { Alive } \\
\end{array}$ & $\begin{array}{l}23 \\
17 \\
\end{array}$ & $\begin{array}{l}57.5 \\
42.5 \\
\end{array}$ & $\begin{array}{c}5 \\
97 \\
\end{array}$ & $\begin{array}{l}4.90 \\
95.1 \\
\end{array}$ & $\begin{array}{c}\mathrm{X}^{2} \\
50.2\end{array}$ & $0.001 * *$ \\
\hline $\begin{array}{l}\text { Hospital stay /days } \\
\text { Mean } \pm \text { SD }\end{array}$ & \multicolumn{2}{|c|}{$21.6 \pm 3.52$} & \multicolumn{2}{|c|}{$3.84 \pm 1.07$} & $\begin{array}{c}\mathrm{U} \\
9.51\end{array}$ & $0.001 * *$ \\
\hline $\begin{array}{l}\text { O2 Saturation } \\
\text { Mean } \pm S D\end{array}$ & \multicolumn{2}{|c|}{$82.3 \pm 10.1$} & \multicolumn{2}{|c|}{$90.6 \pm 4.02$} & $\begin{array}{c}\mathrm{U} \\
6.25\end{array}$ & $0.001 * *$ \\
\hline
\end{tabular}

U: Mann Whitney test FE: Fisher exact test *Significant **High significant 
Table (VII): Univariate and multivariate regression analysis for detection of predictors for need for mechanical ventilation

\begin{tabular}{|c|c|c|c|c|c|c|}
\hline \multirow[t]{2}{*}{ Studied variables } & \multicolumn{2}{|c|}{ Univariate regression } & \multirow[t]{2}{*}{$P$ value } & \multicolumn{2}{|c|}{$\begin{array}{c}\text { Multivariate } \\
\text { regression }\end{array}$} & \multirow[t]{2}{*}{$P$ value } \\
\hline & OR & $95 \% \mathrm{CI}$ & & OR & $95 \% \mathrm{CI}$ & \\
\hline Age / years & 1.46 & $1.23-2.23$ & $0.001 * *$ & 0.982 & $0.936-0.983$ & 0.237 \\
\hline Diabetes mellitus & 2.23 & $1.10-4.53$ & $0.025 *$ & 0.817 & $0.248-2.69$ & 0.741 \\
\hline Dyspnea & 1.03 & $1.04-1.06$ & 0.998 & - & - & - \\
\hline Cyanosis & 12.7 & $5.48-29.5$ & $0.001 * *$ & 1.02 & $0.606-2.82$ & 0.187 \\
\hline CT score & 0.225 & $0.120-0.424$ & $0.001 * *$ & 0.303 & $0.118-0.776$ & $0.013 *$ \\
\hline O2 Saturation & 1.43 & $1.26-1.62$ & $0.001 * *$ & 1.25 & $1.03-1.53$ & $0.023 *$ \\
\hline Bacterial infection & 3.41 & $1.23-5.67$ & $0.001 * *$ & 2.38 & $2.01-4.63$ & $0.001 * *$ \\
\hline
\end{tabular}

OR: Odds ratio CI: Confidence interval
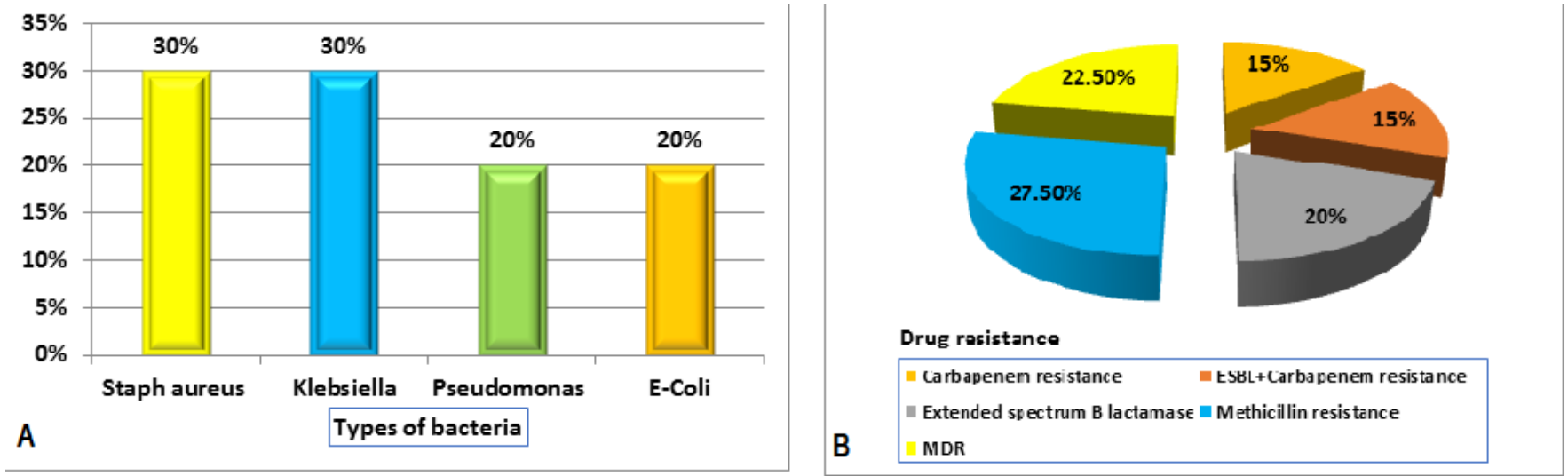

Figure (2): a- Distribution of types of bacteria among positive culture patients. b- Distribution of drug resistance among bacterial isolates.

\section{Discussion}

In this study, percentage of infected males was more than females, coincided with multiples studies as shown in met analysis study of $\mathbf{L i}$ et al. (9). This could be clarified by the high expression of angiotensin-converting enzyme-2 (ACE 2; receptors for coronavirus) in male than female, immunological factors driven by sex hormone and $\mathrm{X}$ chromosome and also gender behaviour (lifestyle) Bwire, (10). Diabetes mellitus and hypertension were the commonest comorbidities reported that agreed with previous studies Yang et al., (11) \& Huang et al., (12) \& Liu et al., (13). Our results are in line with previous studies shown in meta-analysis of Li et al. (9) and Yang et al. , (11), we found that fever, dyspnoea and cough, were the most common presenting symptoms, while GIT symptoms were less common.

In this current study, there was a highly significant difference between mechanically ventilated and non-ventilated patients regarding age together with positive correlation between age and hospital stay and a negative correlation between age and $\mathrm{O}_{2}$ saturation. This was in line with multiple studies showed that older age was the strongest risk factor associated with hospitalization, mechanical ventilation, and mortality (14), (15), (16).

The defects in T-cell and B-cell function with age together with extra production of type 2 cytokines could lead to decrease in the control of viral replication and more extended pro-inflammatory 
responses that lead to high infection rate $\&$ poor outcome (17). More than $50 \%$ of diabetic patients were admitted in ICU with a highly significant difference. Multiple studies showed that diabetes mellitus increases the risk of infection, together with the rates of intensive care unit (ICU) admission, respiratory support, and mortality (18), (15).This could be explained by the advanced risk of severe pneumonia, the higher levels of enzymes linked to tissue injury, hysterical inflammatory response, increased level of inflammatory markers, and hypercoagulable state in diabetic compared to non-diabetic patients, suggesting that diabetes increases the susceptibility to an inflammatory storm that worsen COVID-19 (19).

Suleyman et al (20) found that dyspnea and hypoxia at presentation was associated with hospitalization and the need for ICU management. However, fever and headache at presentation were more in patients presented in the general practical unit than patients in the ICU on univariable analysis. Gastrointestinal symptoms were presenting symptoms in a substantial proportion of patients required hospitalization, similar to the data reported in the COVID-19-Associated Hospitalization Surveillance Network. Also, in the initial reports from Wuhan, China, during the pandemic; dyspnea was recorded in 54\% of patients and was linked to complex end point of admission to an ICU, mechanical ventilation management, and death (21). A similar prevalence of dyspnea was recorded in 21 critically ill patients in Washington State and in the COVID-19Associated Hospitalization Surveillance Network database (22), (23).

Multiple studies were in line with this current study regarding chest CT finding. Guan et al (24) found that the most common patterns on CT chest were ground-glass opacity (56.4\%) and bilateral patchy shadowing (51.8\%). The median duration of hospitalization was 12.0 days (mean 12.8).Also, the study of Ramadan et al (25) that reported that the frequency of bilateral peripheral multilobar ground-glass opacities was the characteristic CT patterns in COVID-19 infection as reported by systematic review done by (26).

Li et al , (27) found that consolidation was significantly more common in severe and critical patients in comparison with ordinary groups and the CT scores of the severe and critical patients were significantly higher than those of the ordinary patients $(P<0.001)$. This shows that the alveoli are completely full with inflammatory exudate which means that the virus diffuses into the respiratory epithelium, ending in necrotizing bronchitis, extensive alveolar damage and finally death(28).

The blood culture results and the characteristics of COVID-19 patients vary greatly between centers and geographic sites (5). Among COVID19 patients we detected an increased incidence of hospital-acquired BSIs, mostly due to multidrugresistant pathogens. Our results match studies conducted by Kokkoris et al.(29) and Giacobbe et al. (30) studies with $40 \%$ and $\geq 25 \%$ respectively for hospital-acquired BSIs.

In current study Blood stream infections caused by Methicillin resistant Staphylococci (MRSA) were represented by $27.5 \%$ while MDR Gram negative bacilli recorded $72.5 \%$. Other studies (31) had limited their analysis only to the first $48 \mathrm{~h}$ of hospital admission \& reported lower BSI rate than ours. However our data are in agreement with Wang et al.(32) with 20\% for MRSA and 80\% for MDR Gram negative bacilli. Also Bogossian et 
al.(33) reported BSI rates with $10 \%$ for MRSA, $77 \%$ for MDR Gram negative bacilli (29\% for ESBL and $48 \%$ for MBL producers). Old age, Hypertension, Diabetes, Assisted ventilation with prolonged hospital stay were significant risk factors for BSI in COVID 19 patients as shown in different researches $(3,6,32)$.

The COVID 19 infection \& mortality rates are multifactorial issues that reflect; COVID strain virulence and infectivity, demographic charactersassociated infections and presenting co-morbidities of patients as well as availabilities of health care personnel-protocols and facilities. The rates vary significantly between different countries (2). All previously mentioned factors could explain morbidity \& mortality rates reported in this study especially as more than $80 \%$ of ventilated cases with MDR blood stream infections died during hospitalization.

\section{Conclusion}

Egypt is among the countries that reported high rates of antimicrobial resistance. In our study all patients had taken empirical antibiotics. All blood stream bacterial pathogens were multidrug resistant. Infections were detected among old aged diabetic, hypertensive and mechanically ventilated patients. High CT score, decreased O2 saturation and presence of blood stream bacterial infection are independent predictors for need of mechanical ventilation among severe \& critical COVID 19 cases. Prescribing antibiotics in COVID patients should be guided by careful clinical and laboratory assessment to improve their management and outcomes.

There was no funding for this study. Conflict of interest
The authors declare that there are no conflicts of interest.

\section{Ethical approval}

Prior to sample collection, written approval agreed by the Human Rights Committee in Research at Menoufia University was obtained from all studied cases and controls.

\section{References}

1- Haque A, Pant AB. Efforts at COVID-19 Vaccine Development: Challenges and Successes. Vaccines. 2020; 8(4):739. https://doi.org/10.3390/vaccines8040739

2- World Health Organization. (2021). Global surveillance for COVID-19 caused by human infection with COVID-19 virus: interim guidance, March 2021. World Health Organization. https://apps.who.int/iris/handle/10665/331 506. License: CC BY-NC-SA 3.0 IGO

3- European Centre for Disease Prevention and Control. COVID-19 situation update worldwide, as of 25 May $2020 \quad$ [Internet]. 2020 (https://www.ecdc.europa.eu/en/geographi cal-distribution-2019-ncov-cases.

4- Morens DM, Daszak P, Taubenberger JK. Escaping Pandora's Box - Another Novel Coronavirus. N Engl J Med. 2020; 382(14):1293-1295. doi:10.1056/NEJMp2002106

5- Yu D, Ininbergs K, Hedman K, Giske CG, Strålin K, Özenci V (2020) Low prevalence of bloodstream infection and high blood culture contamination rates in patients with COVID-19. PLoS ONE 15(11):

e0242533. 
https://doi.org/10.1371/journal.pone.02425 33

6- Bassetti M, Kollef MH, Timsit JF. Bacterial and fungal superinfections in critically ill patients with COVID-19. Intensive Care Med. 2020;46(11):20712074. doi:10.1007/s00134-020-06219-8

7- Prokop M, van Everdingen W, van Rees Vellinga $\mathbf{T}$, et al. CO-RADS: A Categorical CT Assessment Scheme for Patients Suspected of Having COVID-19Definition and Evaluation. Radiology. 2020;296(2):E97-E104. doi:10.1148/radiol.2020201473

8- Clinical and Laboratory Standards Institute (CLSI). Performance standards for antimicrobial susceptibility testing; 31th ed. CLSI supplement M100. Wayne:Clinical and Laboratory Standards Institute; 2021.

9- Li LQ, Huang T, Wang YQ, et al. COVID-19 patients' clinical characteristics, discharge rate, and fatality rate of meta-analysis. J Med Virol. 2020;92(6):577-583. doi:10.1002/jmv.25757

10- Bwire GM. Coronavirus: Why Men are More Vulnerable to Covid-19 Than Women? [published online ahead of print, 2020 Jun 4]. SN ComprClin Med. 2020;13. doi:10.1007/s42399-020-00341-w

11- Yang $\mathbf{J}$, Zheng $\mathbf{Y}$, Gou $\mathbf{X}$, et al. Prevalence of comorbidities and its effects in patients infected with SARS-CoV-2: a systematic review and meta-analysis. Int $\mathrm{J}$ Infect Dis. 2020;94:91-95. doi:10.1016/j.ijid.2020.03.017
12- Huang C, Wang Y, Li X, et al. Clinical features of patients infected with 2019 novel coronavirus in Wuhan, China [published correction appears in Lancet. 2020 Jan 30;:]. Lancet. 2020;395(10223):497-506. doi:10.1016/S0140-6736(20)30183-5

13- Liu M, Liu SW, Wang LJ, et al. Burden of diabetes, hyperglycaemia in China from to 2016: Findings from the 1990 to 2016, global burden of disease study. Diabetes Metab. 2019;45(3):286-293. doi:10.1016/j.diabet.2018.08.008

14- Cummings MJ, Baldwin MR, Abrams D, et al. Epidemiology, clinical course, and outcomes of critically ill adults with COVID-19 in New York City: a prospective cohort study. Lancet. 2020;395(10239):1763-1770. doi:10.1016/S0140-6736(20)31189-2

15- Ioannou GN, Locke E, Green P, et al. Risk Factors for Hospitalization, Mechanical Ventilation, or Death Among 10131 US Veterans With SARS-CoV-2 Infection. JAMA Netw Open. 2020;3(9):e2022310. Published 2020 Sep 1.

doi:10.1001/jamanetworkopen.2020.22310

16- Zhou F, Yu T, Du R, et al. Clinical course and risk factors for mortality of adult inpatients with COVID-19 in Wuhan, China: a retrospective cohort study [published correction appears in Lancet. 2020 Mar 28;395(10229):1038] [published correction appears in Lancet. 2020 Mar 28;395(10229):1038]. Lancet. 
2020;395(10229):1054-1062.

doi:10.1016/S0140-6736(20)30566-3

17- Opal SM, Girard TD, Ely EW. The immunopathogenesis of sepsis in elderly patients. Clin Infect Dis. 2005;41Suppl 7:S504-S512. doi:10.1086/432007

18- Kumar A, Arora A, Sharma P, et al. Is diabetes mellitus associated with mortality and severity of COVID-19? A metaanalysis. Diabetes MetabSyndr. 2020;14(4):535-545.

doi:10.1016/j.dsx.2020.04.044

19- Guo W, Li M, Dong Y, et al. Diabetes is a risk factor for the progression and prognosis of COVID-19 [published online ahead of print, 2020 Mar 31]. Diabetes Metab Res Rev. 2020;e3319. doi:10.1002/dmrr.3319

20- Suleyman G, Fadel RA, Malette KM, et al. Clinical Characteristics and Morbidity Associated With Coronavirus Disease 2019 in a Series of Patients in Metropolitan Detroit. JAMA Netw Open. 2020;3(6):e2012270. Published 2020 Jun 1.

doi:10.1001/jamanetworkopen.2020.12270

21- State of Michigan. COVID-19 response and mitigation strategies for racial and ethnic populations and marginalized communities. Published April 2020. Accessed May 2020. https://www.michigan.gov/documents/md hhs/OEMH_COVID-

19_Response_Mitigation_Strategies_Targ eting_Racial_Ethnic_Populations_Margin alized_Communities_FINAL_689586_7.p df
22- Garg, S., Kim, L., Whitaker, M., O'Halloran, A., Cummings, C., Holstein, R., Prill, M., Chai, S. J., Kirley, P. D., Alden, N. B., Kawasaki, B., Yousey-Hindes, K., Niccolai, L., Anderson, E. J., Openo, K. P., Weigel, A., Monroe, M. L., Ryan, P., Henderson, J., ... Fry, A. (2020). Hospitalization rates and characteristics of patients hospitalized with laboratoryconfirmed coronavirus disease 2019 Covid-net, 14 states, March 1-30, 2020. Morbidity and Mortality Weekly Report, 69(15).

https://doi.org/10.15585/MMWR.MM691 $5 \mathrm{E} 3$

23- Arentz M, Yim E, Klaff $L$, et al. Characteristics and Outcomes of 21 Critically Ill Patients With COVID-19 in Washington State. JAMA. 2020;323(16):1612-1614. doi:10.1001/jama.2020.4326

24- Guan WJ, Ni ZY, Hu Y, et al. Clinical Characteristics of Coronavirus Disease 2019 in China. N Engl J Med. 2020;382(18):1708-1720. doi:10.1056/NEJMoa2002032

25- Ramadan HKA, Mahmoud MA, Aburahma MZ, Elkhawaga AA, ElMokhtar MA, Sayed IM, Hosni A, Hassany SM, Medhat MA. Predictors of Severity and Co-Infection Resistance Profile in COVID-19 Patients: First Report from Upper Egypt. Infect Drug Resist. 2020;13:3409-3422 https://doi.org/10.2147/IDR.S272605 
26- Salehi S, Abedi A, Balakrishnan S,

Gholamrezanezhad A. Coronavirus Disease 2019 (COVID-19): A Systematic Review of Imaging Findings in 919 Patients. AJR Am J Roentgenol. 2020;215(1):87-93. doi:10.2214/AJR.20.23034

27- Li K, Wu J, Wu F, et al. The Clinical and Chest CT Features Associated With Severe and Critical COVID-19 Pneumonia. Invest Radiol. 2020;55(6):327-331. doi:10.1097/RLI.0000000000000672

28- Xu Z, Shi L, Wang Y, et al. Pathological findings of COVID-19 associated with acute respiratory distress syndrome [published correction appears in Lancet Respir Med. 2020 Feb 25;:]. Lancet Respir Med. 2020;8(4):420-422. doi:10.1016/S2213-2600(20)30076-X

29- Kokkoris S, Papachatzakis I, Gavrielatou E, et al. ICU-acquired bloodstream infections in critically ill patients with COVID-19. J Hosp Infect. 2021;107:95-97. doi:10.1016/j.jhin.2020.11.009

30- Giacobbe DR, Battaglini D, Ball L, et al. Bloodstream infections in critically ill patients with COVID-19. Eur J Clin Invest. 2020;50(10):e13319. doi:10.1111/eci.13319

\section{1- Lansbury L, Lim B, Baskaran V, Lim}

WS. Co-infections in people with COVID19: a systematic review and meta-analysis. J Infect. 2020;81(2):266-275. doi:10.1016/j.jinf.2020.05.046
32- Wang L, Amin AK, Khanna P, et al. An observational cohort study of bacterial coinfection and implications for empirical antibiotic therapy in patients presenting with COVID-19 to hospitals in North West London. J AntimicrobChemother. 2021;76(3):796-803. doi:10.1093/jac/dkaa475

33- Bogossian EG, Taccone FS, Izzi A, et al. The Acquisition of Multidrug-Resistant Bacteria in Patients Admitted to COVID19 Intensive Care Units: A Monocentric Retrospective Case Control Study. Microorganisms. 2020;8(11):1821. Published $2020 \quad$ Nov 19. doi:10.3390/microorganisms8111821 